\title{
Position Control of Motion Compensation Cardiac Catheters
}

\author{
Samuel B. Kesner, Member, IEEE, and Robert D. Howe, Senior Member, IEEE
}

\begin{abstract}
Robotic catheters have the potential to revolutionize cardiac surgery by enabling minimally invasive structural repairs within the beating heart. This paper presents an actuated catheter system that compensates for the fast motion of cardiac tissue using 3-D ultrasound image guidance. We describe the design and operation of the mechanical drive system and catheter module and analyze the catheter performance limitations of friction and backlash in detail. To mitigate these limitations, we propose and evaluate mechanical and control-system compensation methods, which include inverse and model-based backlash compensation, to improve the system performance. Finally, in vivo results are presented, which demonstrate that the catheter can track the cardiac tissue motion with less than 1-mm rms error. The ultimate goal of this research is to create a fast and dexterous robotic catheter system that can perform surgery on the delicate structures inside of the beating heart.
\end{abstract}

Index Terms-Heart valves, medical robots, motion compensation, robotic catheters.

\section{INTRODUCTION}

$\mathbf{H}$ EART disease is the leading cause of death in most industrialized nations [1]. Physicians and engineers are involved in the development of a myriad of new procedures, drugs, and technologies to treat ailments that can affect the health and function of the human heart. One of the most significant advances in cardiac therapies is the usage of cardiac catheters to give clinicians direct access to the beating heart via the vascular system. This enables diagnosis and treatment without the use of highly invasive open-heart surgical techniques.

Cardiac catheters are long and thin flexible tubes and wires that are inserted into the vascular system and passed into the heart. Innovations in catheter technology have greatly expanded the range of procedures that interventional cardiologists can perform inside the heart using minimally invasive techniques. Procedures that are now performed using catheters include measuring cardiac physiological function, dilating vessels and valves,

Manuscript received October 28, 2010; revised April 1, 2011; accepted June 16, 2011. Date of publication July 22, 2011; date of current version December 8, 2011. This paper was recommended for publication by Associate Editor G. Oriolo and Editor Y. Choi upon evaluation of the reviewers' comments. This work was supported by the U.S. National Institutes of Health under Grant NIH R01 HL073647.

S. B. Kesner is with the Harvard School of Engineering and Applied Sciences, Cambridge, MA, 02138 USA (e-mal: skesner@seas.harvard.edu).

R. D. Howe is with the Harvard School of Engineering and Applied Sciences, Cambridge, MA, 02138 USA, and also with the Harvard Massachusetts Institute of Technology Division of Health Sciences and Technology, Cambridge, MA, 02139 USA (e-mail: howe@ seas.harvard.edu).

Color versions of one or more of the figures in this paper are available online at http://ieeexplore.ieee.org.

Digital Object Identifier 10.1109/TRO.2011.2160467 and implanting prosthetics and devices [2]. While catheters can perform many tasks inside the heart, they do not yet allow clinicians to interact with heart tissue with the same level of skill as in open heart surgery. A primary reason for this is that current catheters do not have the dexterity, speed, and force capabilities to perform complex tissue modifications on moving cardiac tissue.

Robotic catheters are a potential solution to these limitations. Current robotic cardiac catheters, such as the commercially available Artisan Control Catheter (Hansen Medical, Mountain View, CA) or CorPath Vascular Robotic System (Corindus Vascular Robotics, Natick, MA), allow for teleoperated guidance of a catheter tool inside the heart [3]-[6]. These devices permit a human operator to control the positioning of a catheter in vivo. However, these actuated catheter technologies do not provide sufficient speeds to allow the catheters end effectors to keep up with the fast motion of intracardiac structures [7], [8].

Motion compensation is required when operating inside the beating heart because it enables far more dexterous interactions. It also limits the risk of injury from catheter collisions with fast moving tissue structures. Researchers have developed robotic approaches to compensating for the motion of the beating heart [9]-[11], but these techniques are directed at procedures that repair coronary arteries on the external surface of the heart. In a previous work, we have developed robotic devices that compensate for the motion of internal heart structures in vivo with a handheld robotic instrument inserted through incisions in the heart wall [8], [12]-[15]. The motion of the tissue target is tracked in real-time using 3-D ultrasound (3DUS) imaging [13], [14]. This work shows that single degree of freedom (DOF) servoing is sufficient to accurately track the motion of certain cardiac structures, which includes the human mitral valve annulus [7], [15]. The handheld rigid tool approach enables beating heart procedures that alleviate the risks associated with stopped heart techniques [16], but the necessity to create incisions through the chest and into the heart wall requires intubation and deep anesthesia. This means that the rigid tool approach is still relatively invasive.

We propose to apply our successful robotic cardiac motion compensation techniques to catheters in order to minimize invasiveness. In the envisioned clinical system, a drive system at the base end of the catheter will actuate a catheter guidewire inside a flexible sheath (see Fig. 1). The sheath is manually advanced into the heart and positioned by a clinician near the structure of interest. The motion compensation system is then activated. At the distal end of the catheter inside the heart, the guidewire tip then translates in and out of the sheath under ultrasound 


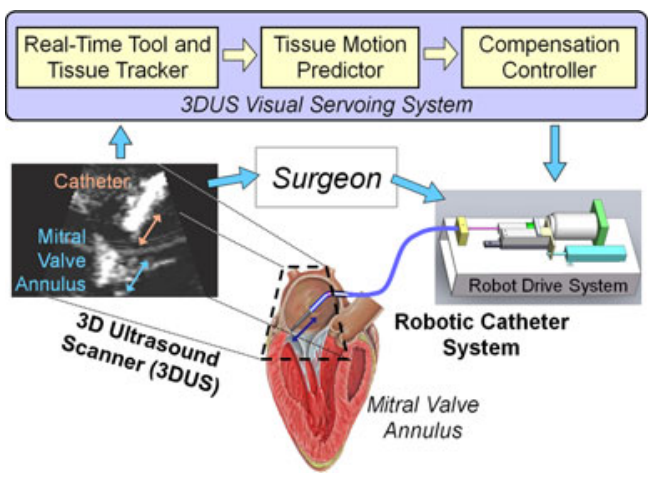

Fig. 1. Robotic catheter system consists of a drive system, a catheter module, and a 3DUS visual servoing system. The system compensates for the fast motion of the cardiac tissue using 3-D ultrasound imaging and a visual servoing system while the surgeon performs the repair procedure.

image guidance to compensate for the movement of the cardiac structures and perform repair.

This paper investigates the design of 3-D ultrasound-guided robotic catheters for beating heart repair. First, we present a novel prototype catheter system and determine its performance limitations. Our preliminary work was the first to identify and characterize the robotic catheter performance limitations under fast servoing, particularly friction and backlash behavior [17]. In this paper, we propose and evaluate mechanical design and control methods to improve the system's trajectory tracking performance by compensating for these friction and backlash effects, which include a new backlash compensation control system. In addition, the system design and control strategies are validated through new in vitro and in vivo experimental results.

\section{SySTEM DESIGN}

The prototype robotic catheter system is designed to compensate for the motion of the outer annulus of the mitral valve, which is the major valve between the left atrium and ventricle. This valve exhibits some of the largest motions and greatest velocities of any structure inside the heart. Our previous work on compensating for the mitral valve annulus has shown that the motion is primarily along one axis of motion; thus, a single DOF system can be used to sufficiently compensate for the valve motion [7].

The actuated catheter system performance parameters were derived from human mitral valve physiology values [7], [15] The principal functional requirements are a single actuated linear degree of freedom with at least $20 \mathrm{~mm}$ of travel and velocity and acceleration of at least $210 \mathrm{~mm} / \mathrm{s}$ and $3800 \mathrm{~mm} / \mathrm{s}^{2}$, respectively. The catheter components should have the same dimensions and materials as current clinical cardiac catheters. Finally, the system should be able to apply a sufficient force to modify cardiac tissue, approximately $4 \mathrm{~N}$.

The system can be divided into three main modules: the drive system that actuates the catheter, the catheter module that is inserted into the heart, and the 3-D ultrasound visual servoing system that tracks the tissue and commands the catheter to follow the motion. A user control interface will also be required for

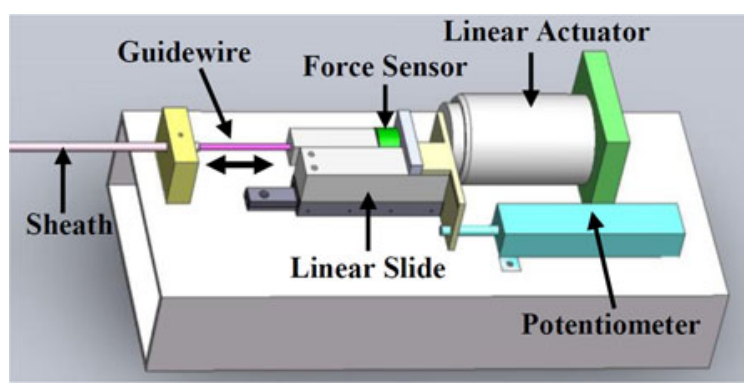

Fig. 2. Catheter drive system consists of a linear actuator, slide, potentiometer, and a force sensor to evaluate the friction on the catheter guidewire. The system servos the guidewire inside the fixed sheath.

TABLE I

EXPERIMENTAL CATHETER DIMENSIONS

\begin{tabular}{ccc}
\hline \hline Sheath Inner Diameter & Guidewire Diameter & Gap Size $(G)$ \\
\hline $1.59 \mathrm{~mm}$ & $0.76 \mathrm{~mm}$ & $0.83 \mathrm{~mm}$ \\
$1.59 \mathrm{~mm}$ & $1.50 \mathrm{~mm}$ & $0.09 \mathrm{~mm}$ \\
$2.38 \mathrm{~mm}$ & $1.50 \mathrm{~mm}$ & $0.88 \mathrm{~mm}$ \\
$2.38 \mathrm{~mm}$ & $2.23 \mathrm{~mm}$ & $0.15 \mathrm{~mm}$ \\
\hline \hline
\end{tabular}

clinical use, which is provided in this prototype by the image processing and control computer.

\section{A. Drive System}

The drive system used in this study (see Fig. 2) is composed of a linear voice coil actuator (NCC20-18-02-1X, H2 W Technologies Inc, Valencia CA; 50.8-mm travel, $26.7 \mathrm{~N}$ peak force), a linear ball bearing slide (BX3-3, Tusk Direct, Inc., Bethel $\mathrm{CT}$ ), and a linear potentiometer position sensor (LP-50F, Midori America Corp, Fullerton CA, linearity: $\pm 0.5 \%$ ). In addition, a force sensor (LCFD-1KG, Omega Engineering, Stamford CT; range: $10 \mathrm{~N}$, accuracy: $+/-0.015 \mathrm{~N}$ ) measures the catheter friction for evaluation purposes.

\section{B. Catheter Module}

The catheter module consists of a sheath, a guidewire, and the end effectors required for each specific repair procedure. The sheath is an $85-\mathrm{cm}$-long section of flexible Teflon tubing that encloses the guidewire, which is a close-wound stainless steel spring that is easily bent but can apply significant compressive forces without buckling. During the procedure, the sheath is inserted from a peripheral blood vessel (typically the femoral vein) into the heart and then fixed in place while the drive system servos the guidewire inside the sheath to compensate for the heart motion. A geometric description of the various combinations of sheaths and guidewires used in this study is detailed in Table I. The gap $G$, which is defined as the difference between the guidewire outer diameter and the sheath inner diameter (see Fig. 3), is a major determinant of system performance, as shown in the following. 


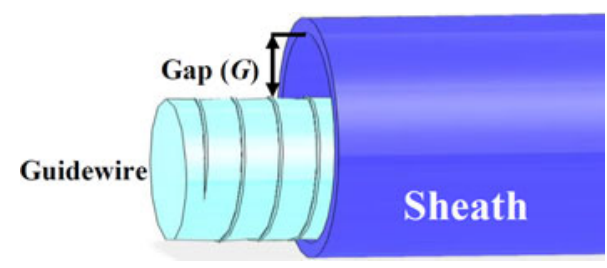

Fig. 3. Catheter guidewire emerging from a sheath. The distance between the outer diameter of the guidewire and the inner diameter of the sheath is defined as the gap size $(G)$.

\section{3-D Ultrasound Visual Servoing System}

The ultrasound servoing system streams 3-D image volumes from the ultrasound scanner to an image processing computer via Ethernet (see Fig. 1). A graphics processing unit (GPU)based radon transform algorithm finds the catheter axis in real time. The target tissue is then located by the projection of the axis forward through the image volume until tissue is encountered; this allows the clinician to designate the target to be tracked by simply pointing at it with the catheter. To compensate for the 50 $100 \mathrm{~ms}$ delay in image acquisition and processing, an extended Kalman filter (EKF) estimates the current tissue location that is based on a Fourier decomposition of the cardiac cycle. Previous in vivo experiments that use this servoing system showed that a rigid instrument system was capable of accurate tracking within the heart, with an rms error of $1.0 \mathrm{~mm}$. See [12]-[15] for a detailed description of the 3DUS visual servoing system.

A PID control system running at $1 \mathrm{kHz}$ controls the position of the linear actuator in the drive system. Commands to the linear actuator are amplified by a bipolar voltage-to-current power supply (BOP 36-12M, Kepco Inc., Flushing NY).

\section{Clinician Controls}

The catheter device automatically compensates for the fast motion of the cardiac tissue, thus allowing the clinician to operate on a "virtually stationary" tissue structure. The procedure is then performed by the interventional cardiologist or surgeon. In the case of the single DOF mitral valve repair, catheter motions in lateral directions (i.e., not in the direction of fast tissue motion) are manually controlled by the usage of conventional catheter controls to bend or rotate the catheter and sheath. To adjust the position of the actuated guidewire in the fast motion direction, clinician commands from a linear joystick are superimposed on the motion compensation trajectory. This allows the clinician to move the guidewire closer to the tissue and perform a repair such as inserting a staple.

\section{Performance Limitations}

Operation of the actuated catheter system reveals two principal performance limitations: the friction forces on the guidewire and the backlash behavior of the guidewire-sheath system. These two phenomena degrade the trajectory tracking accuracy and response time of the actuated catheter tip. Fig. 4 shows an example of an uncompensated catheter tip inaccurately tracking a desired trajectory.
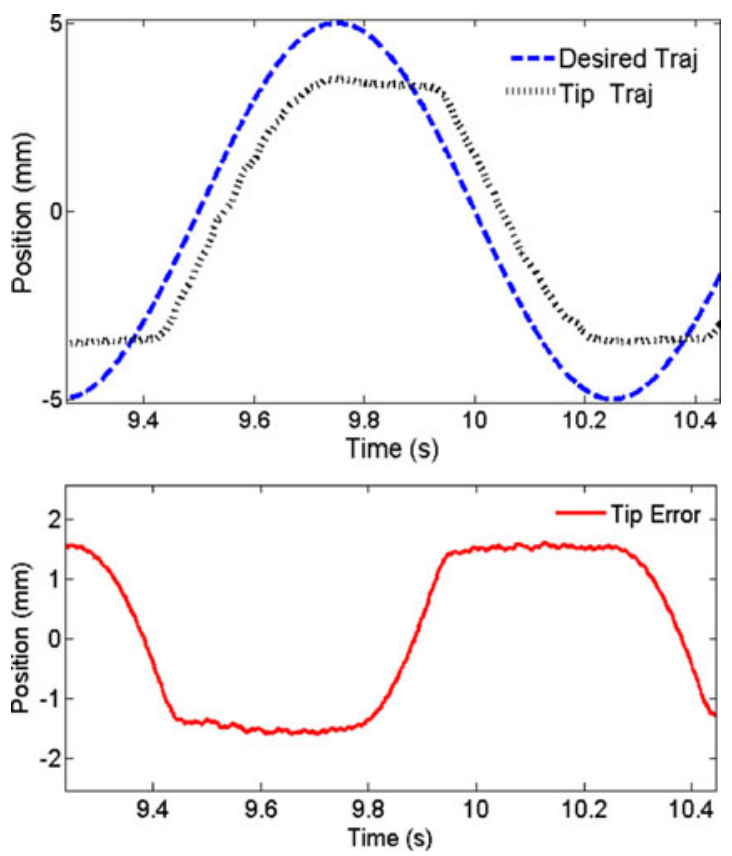

Fig. 4. (Top) Typical catheter tip trajectory tracking accuracy limitations due to friction and backlash. (Bottom) Tip trajectory tracking error.

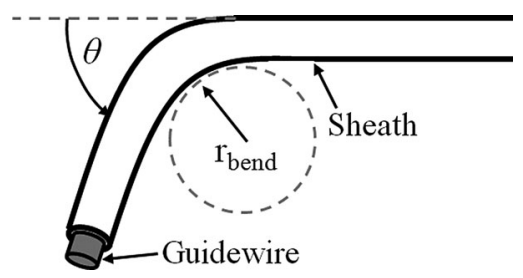

Fig. 5. Catheter sheath configurations used to evaluate the friction and backlash performance limitations.

To determine the major factors that are responsible for these limitations, a parametric study was conducted on the catheter system. The experimental variables examined in this study include the gap size between the sheath and guidewire (see Fig. 3) and the bending configuration of the catheter, characterized by the bend radii and bend angles of the sheath (see Fig. 5). The catheter material properties and the external forces were held constant.

For evaluation purposes, the friction forces in the catheter system and the catheter tip position were directly measured. The friction forces between the guidewire and actuation mechanism were measured with the small force sensor described earlier connected to a differential amplifier (AM502, Tektronix, Beaverton, OR). The catheter tip position was measured with an ultra-low friction rotary potentiometer (CP-2UTX, Midori America Corp, Fullerton, CA, linearity: $\pm 1 \%$ ). The linear motion of the tip was converted into rotation of the potentiometer through a long, lightweight lever arm that connects the tip of the catheter to the sensor. In a clinical setting, tip position will be measured with an electromagnetic tracker or ultrasound imaging. 


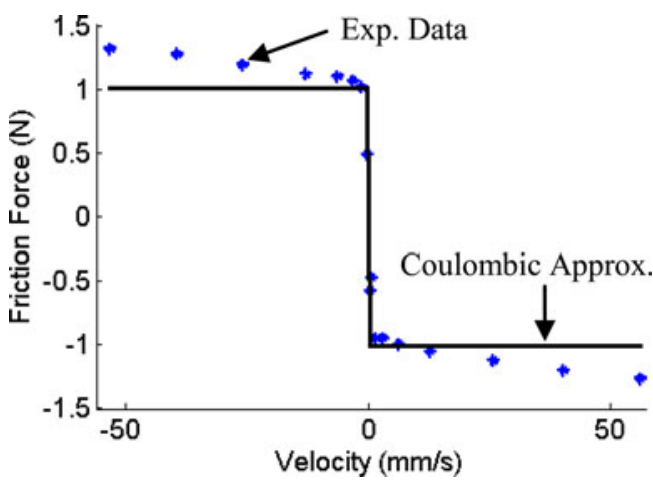

Fig. 6. Catheter friction forces and Coulombic friction approximation as a function of guidewire velocity.

\section{A. Friction}

The first set of experiments examined the catheter system friction as a function of four different sheath-guidewire gap sizes (see Table I), three bending angles $\left(90^{\circ}, 180^{\circ}\right.$, and $\left.360^{\circ}\right)$, and two bend radii $(25$ and $50 \mathrm{~mm}$ ). The sheaths are made of flexible Teflon tubing, and the guidewires are manufactured from uncoated stainless steel. The friction was calculated by commanding a series of constant velocities from the actuator in both the positive and negative directions. Force sensor readings during the constant velocity portion of the trajectory were averaged and plotted against the velocities. The friction data were summarized for each configuration by taking the average of the friction values for each velocity. The data were analyzed with a three-way analysis of variance (ANOVA).

1) Friction Results: Fig. 6 presents a typical frictionvelocity curve for this system. The observed behavior can be approximated as constant dynamic friction plus a component that varies linearly with velocity. For this case, the Coulomb term can be approximated as $1.0 \mathrm{~N}$ of friction and the velocity dependent term as $0.006 \mathrm{~N} /(\mathrm{mm} / \mathrm{s})$. In this study friction is modeled as Coulombic friction because the velocity-dependant contributions are small $(<10 \%)$ for the majority of velocities required to track the heart motion. Configurations with less than $0.05 \mathrm{~N}$ of friction were assumed to be frictionless because the friction was on the order of the sensor drift for the duration of the experiment.

The results of the friction experiments, summarized in Fig. 7, contain a number of trends. The gap size has the strongest influence on guidewire friction $(p<0.0001, F=107.62)$. This parameter directly affects the normal forces applied to the guidewire by the sheath. The normal force is created by any sections of the sheath that might be pinched, locations where the guidewire is constrained to conform to the inner wall of the bending sheath, and places where kinks in the guidewire or sheath cause the two components to come into contact. A small gap size amplifies these issues because smaller deformations in the catheter system cause the sheath and guidewire to interact. Large gap sizes, on the other hand, allow more space for misalignments. Therefore, increasing the gap size decreases the friction that is experienced by the guidewire.
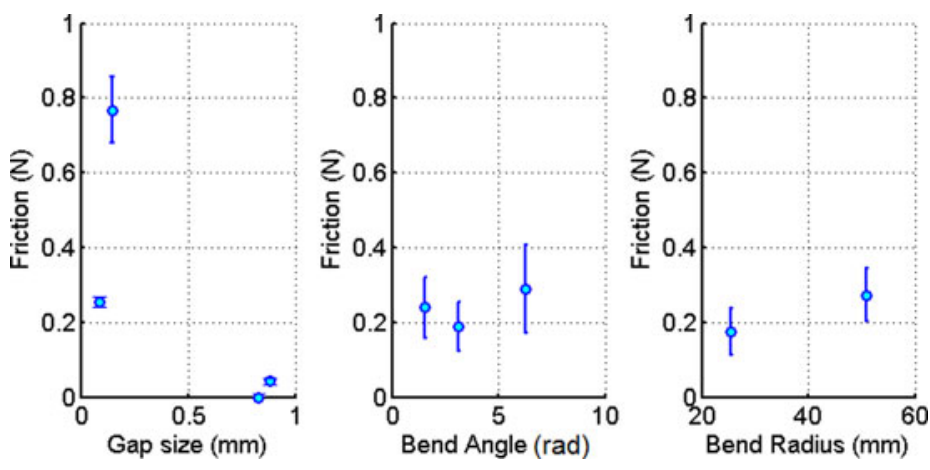

Fig. 7. Friction results as a function of gap size, bend angle, and bend radius. Friction is assumed to be Coulombic, and the symbols are the mean values and bars are the standard error.

The results also show that bend angle has an effect on the friction forces ( $p=0.004, F=6.47$ ). Although the magnitude of the effect is small, it is clearly illustrated when the data are partitioned by gap size as in [17]. One reason for this trend is that bending causes the sheaths' cross sections to deform slightly. This deformation can pinch the guidewire, thus increasing the applied normal forces. In addition, the bending of the sheath forces the inner guidewire to bend in order to conform to the outer sheath. The reaction forces generated by the conforming guidewire increase the normal force and, therefore, the friction on the guidewire.

The bending radii used in this study, which span the typical range for cardiac catheters, do not appear to have a significant impact on the friction measurements $(p=0.64, F=0.23)$.

These results indicate that for certain conditions, only the gap size and catheter bending are required to estimate the friction in the system. However, additional factors that contribute to the total friction that is experienced by the guidewire, including the sheath and guidewire materials and dimensions, the catheter seals and connectors, and the external forces that are applied to the system, complicate the development of a general model of system friction.

\section{B. Backlash}

The backlash properties of the sheath-guidewire system were investigated with the same experimental variables (gap size, bend angle, and bend radius) as the aforementioned friction experiments. The backlash was examined by commanding the base of the catheter system to follow a 1-Hz sinusoidal trajectory (see Fig. 4). This trajectory is a highly simplified version of a mitral valve annulus motion of heart beating at 60 beats $/ \mathrm{min}$. The hysteresis curve for the system plots the input trajectory versus the measured tip position trajectory (see Fig. 8).

The amount of backlash was quantified for each experiment by the width of the backlash hysteresis curve. For example, the hysteresis curve in Fig. 8 has a width of approximately $3 \mathrm{~mm}$. The width of the hysteresis is the amount of displacement commanded at the base of the catheter that does not result in any movement at the tip. The backlash data were analyzed with a three-way ANOVA. 


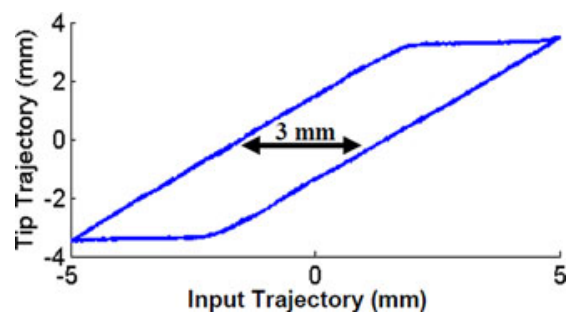

Fig. 8. Hysteresis plot of the trajectory at the drive system versus the catheter tip. The width of this hysteresis curve is referred to as the backlash deadzone, which is equal to $3 \mathrm{~mm}$ in this example.
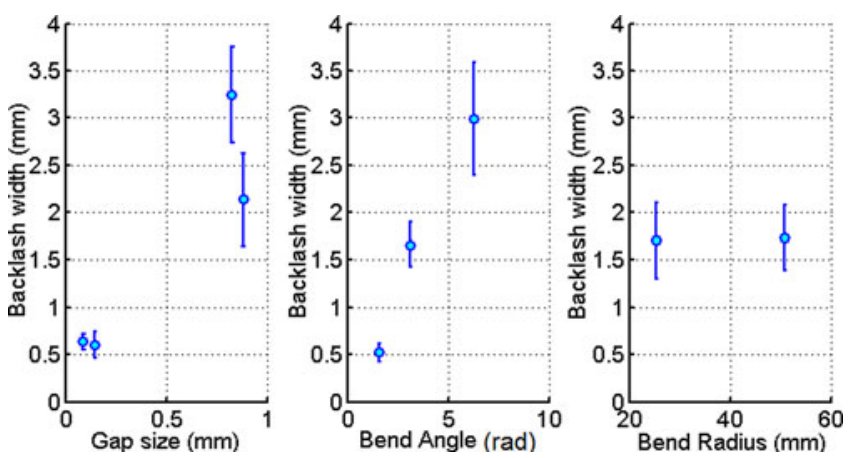

Fig. 9. Backlash results as a function of gap size, bend angle, and bend radius. Symbols are the mean values and bars are the standard error.

1) Backlash Results: The experimental data presented in Fig. 9 summarize the effect of the three experimental parameters on the backlash. Bend angle has the clearest effect on backlash $(p<0.0001, F=28.11)$. The backlash width was found to be approximately proportional to the bend angle. The other parameter that was found to affect the backlash was the gap size $(p<0.0001, F=32.28)$. The data indicate that the larger the gap size, the larger the backlash. Bend radius did not have a significant effect on the backlash width $(p=0.53$, $F=0.41)$.

2) Backlash Model: We developed a model to explain the backlash width values in these experimental results. The catheter guidewires utilized in this system are different from tendon transmission mechanisms because unlike tendons, the guidewires are used both in tension and compression, which can result in buckling [18]-[20]. Unlike backlash models that describe the effects of backlash on displacement and force transmission, our model predicts the size of the backlash deadzone [21].

The model determines the change in length of the guidewire that is required to conform to the curvature inside the catheter sheath. Under tension, the guidewire uses the inside of the curve as a bearing surface and slides along this inner surface of the sheath. When the applied force changes directions to compression, the guidewire is forced to switch positions and conform to the outside of the sheath. This behavior is illustrated in Fig. 10.

As the force $F$ switches from pulling the guidewire in tension to pushing it in compression, the guidewire tip does not initially move, despite the translation of the base because the guidewire must first change positions inside the sheath. The length of the
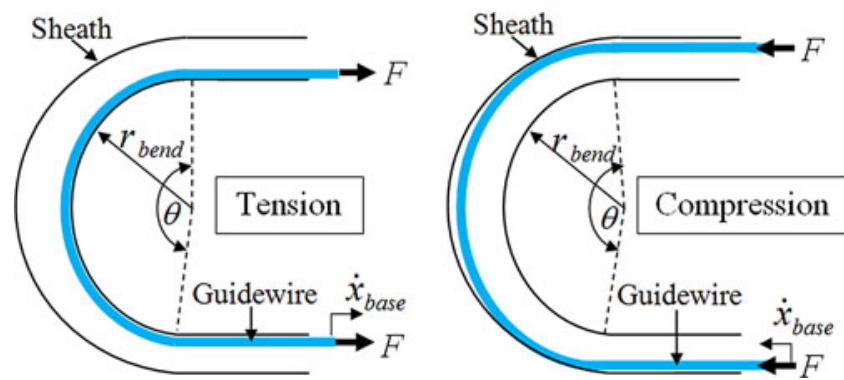

Fig. 10. Guidewire position in the sheath under tension (left) and compression $($ right). Backlash behavior is created by this change of position inside the sheath during transitions from tension to compression.

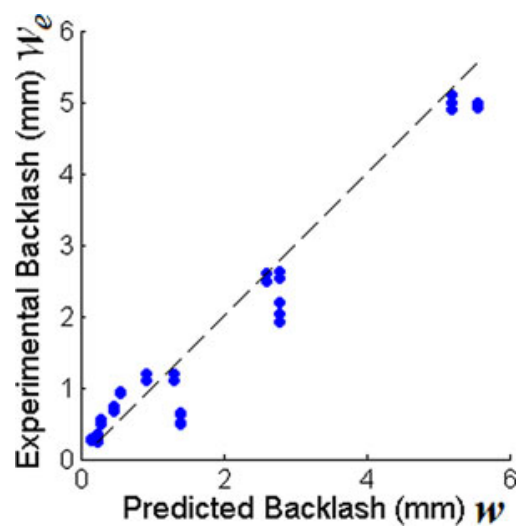

Fig. 11. Model-predicted backlash values versus experimental values. The model agrees with the experimental values with an $r^{2}$ of 0.93 .

guidewire required to change positions depends on the physical configuration and dimensions of the system. The backlash width $w$ can be predicted as the change in curve length

$$
\begin{aligned}
w & =\theta\left(r_{\text {bend }}+D_{\text {sh }}-\frac{1}{2} D_{\text {gw }}\right)-\theta\left(r_{\text {bend }}+\frac{1}{2} D_{\text {gw }}\right) \\
& =\theta\left(D_{\text {sh }}-D_{\text {gw }}\right)
\end{aligned}
$$

where $\theta$ is the total bend angle of the sheath, $r_{\text {bend }}$ is the bend radius of the sheath, $D_{\mathrm{sh}}$ is the inner diameter of the sheath, and $D_{\mathrm{gw}}$ is the diameter of the guidewire (see Fig. 10). The backlash model (1) was evaluated with the backlash data presented in Fig. 9. The model predicted values, $w$, are plotted against the experimental backlash values, $w_{e}$, in Fig. 11. The rms error for the model is $0.4 \mathrm{~mm}$ and the coefficient of determination $r^{2}$ is 0.93 .

The results in Fig. 11 show that the model accurately predicts the backlash width. The model slightly underestimates the backlash for lower backlash values and overestimates for larger values. This trend is most likely caused by the effects of friction on the catheter.

Systems with smaller gap sizes have greater friction, which causes the guidewire to buckle in compression during operation and deforms the outer flexible sheath, thus increasing the backlash width. Systems with larger gaps experience decreased friction forces, which in turn reduce the forces that drive the guidewire to conform to the inner wall of the sheath. An analysis of compliant guidewires buckling inside rigid 


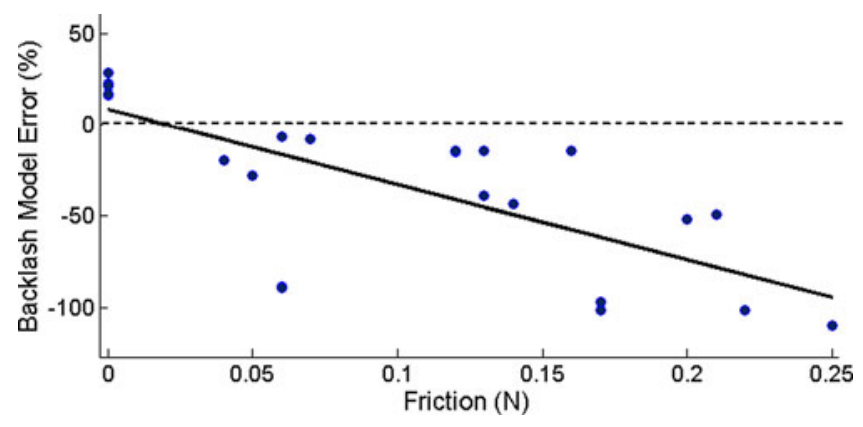

Fig. 12. Backlash model error versus the catheter friction force. The results confirm that the model underestimates the backlash as the friction increases. The coefficient of determination $\left(r^{2}\right)$ for the linear fit is 0.54 .

sheaths was examined in [22], which could be extended to account for the sheath deformation observed here.

3) Backlash-Friction Dependence: The hypothesis that is presented in the previous section is that the catheter friction applies resistance forces to the guidewire that can cause it to deform as it moves, thus increasing the backlash behavior of the catheter tip. To evaluate this hypothesis, a range of normal forces were applied to the sheath at the tip end of the catheter, while the guidewire was driven to follow a sinusoidal trajectory, thus varying the friction level. The sheath configuration was held constant.

The results of this experiment (see Fig. 12) confirm that backlash increases with applied friction, thus causing the model in (1) to further underestimate the backlash. This understanding of how the friction affects backlash can be used to improve backlash compensation.

\section{COMPEnSATION METHODS}

The aforementioned results demonstrate the major factors that affect catheter system trajectory tracking performance. These factors can be used to improve performance through both mechanical design and control system modifications to reduce the impact of friction and backlash on the system.

\section{A. Mechanical Design}

1) Friction: Friction in the catheter system arises from the mechanical rubbing and sticking contacts between the guidewire and the sheath. Friction can be reduced through material selection, material coatings, and lubrication. Catheter sheaths can be made out of plastics that offer both flexibility and lowfriction surfaces, such as polytetrafluoroethylene (PTFE). Clinical guidewires are often coated with low coefficient of friction polymers, such as Teflon, to reduce friction forces. Finally, saline is a possible lubrication method for the catheter system. Current clinical catheter systems use saline to flush air bubbles out of the catheter and prevent blood from backflowing out through the catheter. The saline is also crucial to prevent blood from entering the gap between the guidewire and sheath and coagulating inside the sheath.
2) Backlash: The backlash behavior in the catheter system can be decreased by the reduction of the gap between the guidewire and the sheath. However, reduction of the gap will also increase the friction experienced by the guidewire. This design tradeoff should be considered by selecting the guidewire and sheath with the smallest gap that does not introduce enough friction to significantly increase the backlash width.

\section{B. Control System}

1) Friction: The system backlash and friction can also be reduced through improvements to the control system. For example, feedforward Coulomb friction compensation can be used to reduce the friction force effects in the base module [23]. This method uses a friction predictor that observes the desired catheter velocity and the average friction resistance, and then feeds forward an additional force that the actuator applies to the catheter to compensate for the friction. The feedforward predictor used in this case employs a Coulombic model, which was shown to reasonably approximate the friction forces experienced by the catheter (see Fig. 6).

One limitation of friction compensation is that it primarily improves the trajectory tracking of the drive system module. It is not able to reduce the main source of trajectory tracking error at the catheter tip, the backlash behavior of the guidewire inside the sheath. While backlash is related to friction resistance in the catheter, compensation for friction at the drive system does not reduce the backlash effects on the guidewire.

2) Backlash: An enhanced control system can reduce the backlash behavior by the modification of the trajectory commanded at the base of the catheter. The trajectory can be extended to ensure that the tip of the catheter overcomes the backlash deadzone and reaches the desired location. The general approach is to add an offset $\delta$ to the desired trajectory $x_{d}(t)$ to create a new trajectory for the drive system to follow that will ensure that the tip of the catheter achieves the desired trajectory. The modified trajectory $x_{m}(t)$ can be written as

$$
x_{m}(t)=x_{d}(t)+\delta\left(x_{d}, x_{m}, w\right) .
$$

The offset value $\delta$ can be determined by a number of methods and can vary as a function of the desired trajectory, the previous modified trajectory, the predicted or experimental backlash width, and a range of other system parameters.

Here, we consider two leading trajectory modification control methods, inverse compensation, and model-based compensation.

a) Inverse Compensation: Inverse compensation commands the system to follow a new trajectory that is created by adding the tracking error to the original desired trajectory. This method measures the backlash and uses the inverse value to specify the offset $\delta$ [21]. Fig. 4 presents an example of the tracking error that is caused by backlash in the catheter system. Limitations of this method are that it assumes the system is able to traverse the deadzone region instantaneously and that the backlash behavior is constant and not velocity dependent [21]. Another challenge with this method is that it requires knowledge of the error before the trajectory can be modified, 


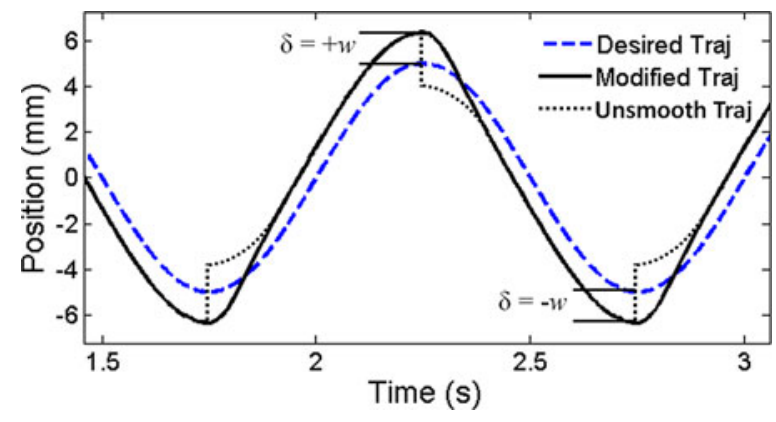

Fig. 13. Desired sinusoidal trajectory and the modified trajectory created with the model-based backlash compensation method. Note the smoothed and unsmoothed transitions between positive and negative offset.

which requires initially running the system without compensation.

b) Model-Based Compensation: Another backlash compensation method is to use the backlash model prediction in (1) to adjust the desired trajectory. Given a known gap size and sheath bend configuration, this model-based controller can estimate the backlash width and then feedforward a trajectory correction to the drive system controller. This method has the advantage that it can adjust the compensation in real time as the bend configuration changes. The sheath configuration measurement can be updated either through imaging or mechanical sensors as the catheter position changes during the procedure.

For this control method, the offset value $\delta$ is a function of the desired and modified trajectories, the width of the backlash deadzone region $w$ calculated with the model in (1), and a smoothing term $\tau$ :

$$
\delta=\left\{\begin{array}{c}
+w-\tau \\
-w+\tau
\end{array}\right.
$$

The sign of the offset is determined by which side of the deadzone the model predicts the catheter tip should be commanded to travel. The additional term $\tau$ is included to smooth the transition of the offset when the desired trajectory requires that the catheter to travel to the other side of the deadzone. Without this smoothing term, the catheter tip would attempt to instantaneously traverse the deadzone and potentially overshoot.

A gradual, smooth transition can be achieved if a transition term $\tau$ is included to modify the backlash offset:

$$
\tau=2 w\left(1-e^{(-\Delta x / G)}\right)
$$

where $\Delta x$ is the distance traveled from the previous side of the backlash deadzone, and $G$ is a gain value used to select how quickly the offset travels across the deadzone. $\tau$ is determined based on the system bandwidth to allow the catheter to transition as fast as possible without causing any significant overshoot. Fig. 13 presents an example of the modified trajectory that is calculated for a given backlash width and a sinusoidal desired trajectory both with and without the smoothing term.

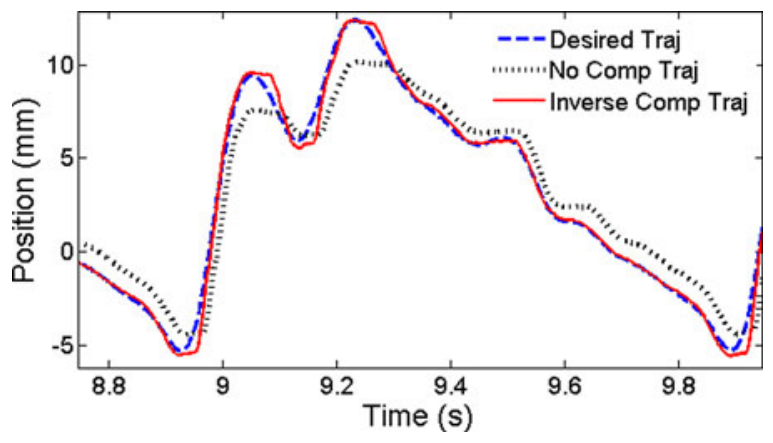

Fig. 14. Recorded human mitral valve annulus trajectory, the tip trajectory, and the inverse compensation improved tip trajectory.

\section{Compensation Method Evaluation}

Backlash and friction compensation are required to improve the catheter system trajectory tracking accuracy. Both inverse and model-based deadzone compensation were tested. A feedforward Coulombic friction compensator was used in addition to these methods. This compensator's primary function is to ensure that the drive system overcomes the friction resistance and accurately follows the desired trajectory.

\section{A. Inverse Compensation}

The inverse compensation method was evaluated on the actuated catheter system in conditions that simulated a cardiac intervention. All of the trajectories tracking evaluations were longer than $10 \mathrm{~s}$ in duration. In this experiment, a $0.76-\mathrm{mm}$ diameter guidewire and a $1.59-\mathrm{mm}$ inner diameter sheath were constrained to a configuration with two $90^{\circ}$ bends that simulated a realistic anatomical approach to pass the catheter from the inferior vena cava into the right atrium with a $50-\mathrm{mm}$ bending radius, crossing the atrial septum, and then turning toward the mitral valve with a $25-\mathrm{mm}$ bend radius. A rubber seal attached to the end of the sheath simulated a seal used to prevent the gap between the sheath and guidewire from filling with blood.

Inverse compensation was first applied to the $1-\mathrm{Hz}$ sinusoidal trajectory. Initially, the tip position trajectory tracking mean absolute error (MAE) for the sinusoidal trajectory was $1.28 \mathrm{~mm}$. The inverse compensation trajectory improved the tip position trajectory tracking by $80 \%$ to an MAE of $0.26 \mathrm{~mm}$.

The compensation method was applied to a typical mitral valve annulus trajectory taken from human ultrasound data [7] (see Fig. 14). Without compensation, the catheter tip failed to track the extremes of the mitral valve trajectory. However, the tip trajectory tracking greatly improved when the inverse compensation trajectory was applied to the system (see Fig. 14). The inverse method reduced the mean absolute error from 1.19 to $0.24 \mathrm{~mm}$ : an improvement of almost $80 \%$.

\section{B. Model-Based Compensation}

The model-based deadzone compensation method was tested with a 1.5 -mm guidewire and a $2.38-\mathrm{mm}$ inner diameter sheath. The sheath was configured to a $180^{\circ}$ bend with an approximately 


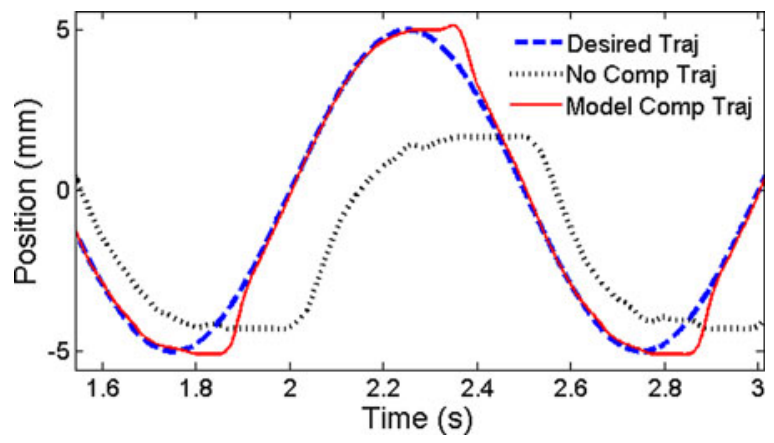

Fig. 15. Sinusoidal trajectory, the tip trajectory, and improved tip trajectory with model-based compensation.

$50-\mathrm{mm}$ bend radius, which is similar to the inverse compensation evaluation experiment. These values were applied to the model in (1) to predict the width of the backlash region. Each evaluation trial was longer than $10 \mathrm{~s}$ in duration.

The results presented in Fig. 15 show that this compensation method greatly improved the catheter trajectory tracking. For tracking a sinusoidal trajectory, the MAE without compensation was $2.34 \mathrm{~mm}$ and the MAE with model-based compensation was $0.24 \mathrm{~mm}$ : an improvement of almost $90 \%$.

\section{Compensation Methods Discussion}

The two backlash compensation methods presented here improve the catheter tip trajectory tracking. One limitation of inverse compensation is that it requires the system to first follow the commanded trajectory inaccurately and then calculate how to alter the trajectory to improve tracking. This approach is impractical for the real-time control because it assumes that backlash is constant during operation, which is not that case when the catheter bend angle and radius change during a procedure. The model-based method, on the other hand, only requires an accurate knowledge of the sheath configuration, which can be found through fluoroscopic imaging or sensors that are embedded in the catheter sheath. Furthermore, the sheath should not require regular readjustment once the catheter is inside the heart during the procedure. Therefore, the model-based approach is a more appropriate compensation method for the clinical setting.

\section{In Vivo VALIDATION}

To investigate the clinical feasibility of image-based catheter control, we integrated the actuated catheter system with the ultrasound visual servoing system that was developed in previous work [7], [12]-[15] and evaluated it in vivo. Controlling a catheter to follow the motion of internal cardiac structures requires real-time sensing of both the catheter tip and tissue target positions. Three-dimensional ultrasound must be used for guidance because it is currently the only real-time volumetric imaging technique that can image tissue through blood. In our original image guidance system, the tip of a hand-held instrument with a rigid shaft was introduced through a small incision in the heart wall. The instrument successfully demonstrated in

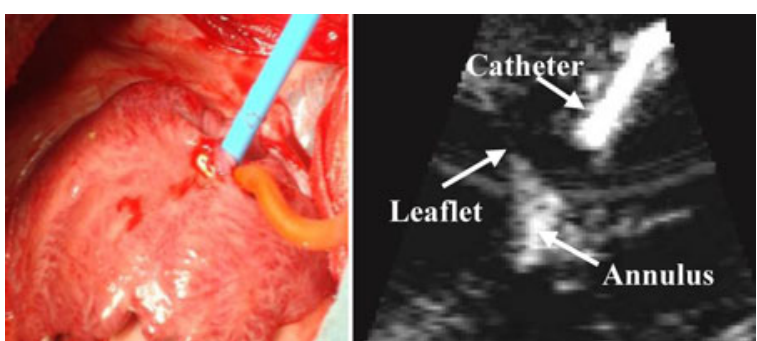

Fig. 16. (Left) Catheter tool inserted into the left atrium. (Right) Ultrasound image showing catheter, mitral valve annulus, and mitral valve leaflets.

vivo the ability to track the tissue motion, control the interaction forces, and place anchors in the mitral valve annulus [8], [15]. The goal of this study is to reduce the invasiveness of this approach by performing these tasks with a catheter.

The image guidance system was evaluated in vivo on a $75 \mathrm{Kg}$ porcine animal model. For this initial study, the actuated catheter was inserted into the beating heart via the top of the left atrium rather than the vasculature to give the surgeon easy access to the mitral valve. The 3-D ultrasound scanner probe (SONOS 7500, Philips Healthcare, Andover, MA) was placed epicardially. After the catheter was introduced into the heart, the surgeon used the ultrasound image to aim the catheter at the mitral valve annulus. The imaging system was then initialized and tracked the valve motion. See Fig. 16 for an image of the catheter device inserted into the porcine left atrium and a 3DUS image of the catheter in vivo.

The catheter module consisted of a sheath with $1.6 \mathrm{~mm}$ inner diameter and a guidewire with a 1.5-mm outer diameter. During the experimental trials, the sheath was configured external to the heart with two $90^{\circ}$ bends that correspond to the path from the femoral vein into the left atrium. The catheter was positioned inside the left atrium so that the tip was $1-2 \mathrm{~cm}$ from mitral annulus. The catheter controller then performed a calibration routine that estimates the magnitude of the friction force in the system. Next, the image processing routines located the catheter with the Radon transform algorithm and then projected forward to find the tissue target and track its trajectory. An extended Kalman filter is used to remove any delay in the trajectory and interpolate the 3DUS information up to the $1-\mathrm{kHz}$ controller rate [8]. The catheter was then servoed to maintain a constant distance between the catheter tip and the target.

\section{A. Tracking Results}

The catheter system successfully tracked the mitral annulus tissue target. Fig. 16 shows a cross section through a typical ultrasound image volume that contains the catheter, mitral valve annulus, and edge of the valve leaflet. Friction compensation was used in this experiment; however, active deadzone compensation was not required because the mechanical design of the catheter system, which includes the selection of a guidewire and sheath with a small gap size, minimized the deadzone.

Fig. 17 shows a plot of the typical catheter tip trajectory and the position of the mitral valve annulus. This plot was generated 

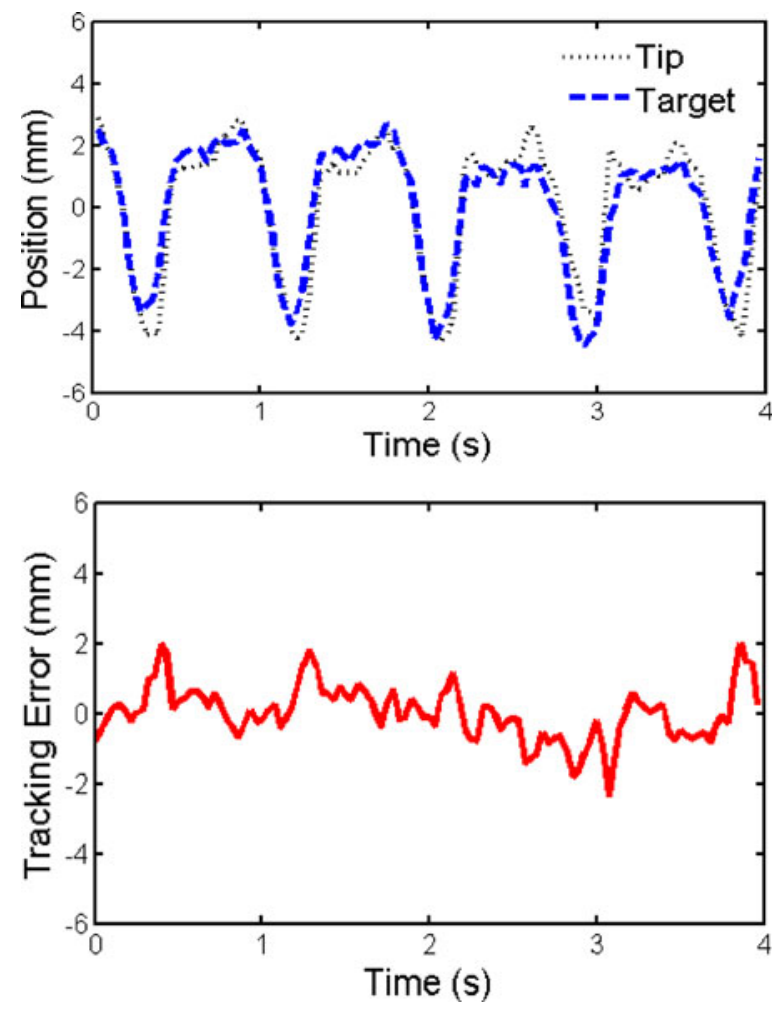

Fig. 17. (Top) Trajectory of the catheter tip and the mitral valve annulus found by manual segmentation. (Bottom) Catheter trajectory tracking error. RMS tracking error was $0.77 \mathrm{~mm}$.

by manually segmenting the position of the catheter tip and valve structure from the 3DUS volumes three times and then averaging the values. The standard deviations of the segmented tip positions were less than $0.22 \mathrm{~mm}$ and the standard deviations of the segmented mitral valve annulus positions were less than $0.32 \mathrm{~mm}$. Because of the seals required to prevent backflow of blood out of the heart and contain the saline in the sheath, friction compensation values as high as $2 \mathrm{~N}$ were required for these experiments.

The image-guided catheter tracked the valve motion with rms errors less that $1.0 \mathrm{~mm}$ in all experimental trials. The duration of each trial was greater than $15 \mathrm{~s}$. The rms error for the trial presented in Fig. 17 is $0.77 \mathrm{~mm}$. The tracking error, which is shown in Fig. 17, was caused by respiration motion not captured in the tissue-tracking system, performance limitations of the actuated catheter caused by backlash and friction, and the small beat-to-beat variations in the valve motion that were not compensated by the image-tracking system. For comparison, the rms tracking error for the catheter system without the compensation controller was over $8 \mathrm{~mm}$ due to the substantial catheter friction.

\section{DISCUSSION}

This work demonstrates that robotic catheters can achieve the speed and tip position control required for intracardiac repair applications such as mitral valve annuloplasty. In addition, catheter position can be accurately controlled by the usage of real-time image guidance in vivo. Porcine in vivo studies achieved excellent tracking results, with $\mathrm{rms}$ errors of less than $1 \mathrm{~mm}$. These results suggest that it is feasible to use robotic catheters to enable new intracardiac repairs that are both minimally invasive and avoid the risks of stopped-heart techniques.

The major technological challenges that are explored in this paper are the limitations on precisely controlling a guidewire inside a catheter sheath: friction and backlash. Friction increases as a function of bending angle, but decreases as a function of the gap size between the guidewire and the sheath. The size of the backlash deadzone is dependent on the gap size and the bending angle. These limitations can be mitigated through mechanical design improvements, such as low-friction coatings and reducing the gap size, and control methods, including inverse and model-based backlash compensation.

While this work demonstrates feasibility and identifies the major challenges, a number of areas for improvement remain. The backlash compensation controllers that are presented here assume a static model for the backlash deadzone. The trajectory tracking could be improved by including an adaptive compensator that updates a model of the system backlash that is based on the catheter friction and the tracking performance or a repetitive control system that takes advantage of the periodicity of the cardiac motion [21], [24], [25]. Another strategy is to provide closed-loop control for the catheter tip position by the usage of electromagnetic or image-based tracking.

To the authors' knowledge, the system described here is the first robotic catheter device that can compensate for the fast motion of structures inside the heart. It is interesting to note that this approach is complementary to current commercial catheter robot systems like the Artisan Control Catheter (Hansen Medical, Mountain View, CA). The Hansen Medical catheter system achieves lateral deflection and sheath translation at roughly manual speeds and could be readily combined with the fast guidewire actuation system described here.

\section{A. Extensions}

While this work has demonstrated the potential of robotic catheter systems to enable new beating heart surgical procedures, a number of extensions will expand the range of procedures this technology can accomplish. These advances included actuation in multiple DOF, force control and sensing capabilities, and more complex catheter mechanisms.

1) Multi-DOF Actuation: Additional fast servoed degrees of freedom will allow the catheter to track cardiac tissue with complex 3-D trajectories. Two additional actuated DOF that will allow the catheter to track an arbitrary point at cardiac velocities are bending of the guidewire shaft and twisting of the guidewire tip (see Fig. 18). These additional DOF can be achieved by adding a single or double pair of bending pull wires inside of the guidewire and adding a rotational servo motor at the drive system end of the catheter to twist the guidewire externally.

2) Force Sensing and Control: Dexterous repairs within the heart require the ability to accurately apply forces against tissue 


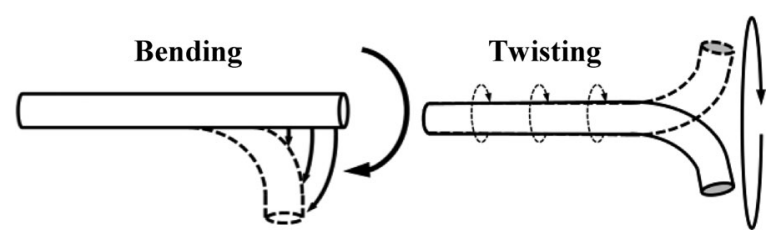

Fig. 18. Additional actuated DOF. (Left) Bending of the catheter tip generated by pull wires inside of the guidewire. (Right) Twisting of the catheter achieved by rotating the guidewire at the drive system module.

targets [26]. This task is made even more challenging because the catheter must interact with quickly moving tissue structures. To this end, we have developed catheter tip force sensors and catheter-specific force control methods [27], [28].

3) Mechanism Development: Additional mechanical mechanisms are also required for the catheter to perform repairs on the inside of the heart. Procedure-specific end-effectors are needed to give the clinician tools to interact with the tissue. For example, in the case of a mitral valve annuloplasty, a suturing or stapling tool is needed to reshape the valve annulus and improve the valve function [15].

A method for bracing the catheter inside the heart will also be required. Without bracing, the catheter sheath will deflect away from the tissue of interest when forces are applied. Bracing will also help the catheter more accurately manipulate the tissue because the system will be fixed relative to cardiac tissue, thus reducing the overall translation distance required for motion compensation [29]-[31].

\section{CONCLUSION}

Robotic catheters have the potential to revolutionize intracardiac procedures by allowing clinicians to perform complicated surgical tasks inside the beating heart without the need for chest incisions, intubation, and deep anesthesia. In this work, friction and backlash were identified as the most significant catheter control limitations, and effective methods to compensate for these limitations were demonstrated. To investigate the feasibility of the usage of image-based catheter servoing to follow the motion of cardiac structures, the system has been integrated with 3 -D ultrasound and an image processing system. In vivo studies showed that excellent tracking can be obtained with rms errors of less than $1 \mathrm{~mm}$. These results demonstrate the feasibility of the usage of robotics catheters to perform minimally invasive intracardiac repairs.

\section{REFERENCES}

[1] A. M. Miniño, M. P. Heron, S. L. Murphy, and K. D. Kochanek, "Deaths: Final data for 2004," National Vital Statistics Reports, vol. 55, no. 19, Aug. 2007, pp. 1-109.

[2] D. S. Baim, Grossman's Cardiac Catheterization, Angiography, and Intervention. Baltimore, MD: Williams \& Wilkins, 2005, p. 992.

[3] T. Fukuda, G. Shuxiang, K. Kosuge, F. Arai, M. Negoro, and K. Nakabayashi, "Micro active catheter system with multi degrees of freedom," in Proc. IEEE Int. Conf. Robot. Automation, May 1994, pp. 2290 2295.

[4] J. Jayender, R. V. Patel, and S. Nikumb, "Robot-assisted catheter insertion using hybrid impedance control," in Proc. IEEE Int. Conf. Robot. Autom., May 2006, pp. 607-612.
[5] D. B. Camarillo, C. F. Milne, C. R. Carlson, M. R. Zinn, and J. K. Salisbury, "Mechanics modeling of tendon-driven continuum manipulators," IEEE Trans. Robot., vol. 24, no. 6, pp. 1262-1273, Dec. 2008.

[6] R. Beyar, "Navigation within the heart and vessels in clinical practice," Ann. NY Academy Sci., vol. 1188, pp. 207-218, 2010.

[7] D. T. Kettler, R. D. Plowes, P. M. Novotny, N. V. Vasilyev, P. J. del Nido, and R. D. Howe, "An active motion compensation instrument for beating heart mitral valve surgery," in Proc. IEEE/RSJ Int. Conf. Intell. Robots Syst., Oct./Nov. 2007, pp. 1290-1295.

[8] S. G. Yuen, S. B. Kesner, N. V. Vasilyev, P. J. del Nido, and R. D. Howe, "3-D ultrasound-guided motion compensation system for beating heart mitral valve repair," in Proc. Med. Image Comput. Comput.-Assisted Intervention, LCNS, vol. 5241, 2008, pp. 711-719.

[9] O. Bebek and M. Cavusoglu, "Intelligent control algorithms for robotic assisted beating heart surgery," IEEE Trans. Robot., vol. 23, no. 3, pp. 468480, Jun. 2007

[10] R. Ginhoux, J. Gangloff, M. de Mathelin, L. Soler, M. M. A. Sanchez, and J. Marescaux, "Active filtering of physiological motion in robotized surgery using predictive control," IEEE Trans. Robot., vol. 21, no. 1, pp. 67-79, Feb. 2005.

[11] Y. Nakamura, K. Kishi, and H. Kawakami, "Heartbeat synchronization for robotic cardiac surgery," in Proc. IEEE Int. Conf. Robot. Autom., 2001, pp. 2014-2019.

[12] S. G. Yuen, P. M. Novotny, and R. D. Howe, "Quasiperiodic predictive filtering for robot-assisted beating heart surgery," in Proc. IEEE Int. Conf. Robot. Autom., May 2008, pp. 3875-3880.

[13] P. M. Novotny, J. A. Stoll, P. E. Dupont, and R. D. Howe, "Real-time visual servoing of a robot using three-dimensional ultrasound," in Proc. IEEE Int. Conf. Robot. Autom., Apr. 2007, pp. 2655-2660.

[14] S. G. Yuen, N. V. Vasilyev, P. J. del Nido, and R. D. Howe, "Robotic tissue tracking for beating heart mitral valve surgery," Med. Image Analysis, 2010, doi: 10.1016/j.media.2010.06.007 (in press).

[15] S. G. Yuen, D. T. Kettler, P. M. Novotny, R. D. Plowes, and R. D. Howe, "Robotic motion compensation for beating heart intracardiac surgery," Int. J. Robot. Res., vol. 28, no. 10, pp. 1355-1372, 2009.

[16] M. F. Newman, J. L. Kirchner, B. Phillips-Bute, V. Gaver, H. Grocott, R. H. Jones, D. B. Mark, J. G. Reves, and J. A. Blumenthal, "Longitudinal assessment of neurocognitive function after coronary-artery bypass surgery," New England J. Med., vol. 344, no. 6, pp. 395-402, 2001

[17] S. B. Kesner and R. D. Howe, "Design and control of motion compensation cardiac catheters," in Proc. IEEE Int. Conf. Robot. Autom., May, 2010, pp. 1059-1065.

[18] M. Kaneko, T. Yamashita, and K. Tanie, "Basic considerations on transmission characteristics for tendon drive robots," in Proc. Fifth Int. Conf. Adv. Robot., 1991, pp. 827-832.

[19] A. Nahvi, J. M. Hollerbach, Y. Xu, and I. W. Hunter, "An investigation of the transmission system of a tendon driven robot hand," in Proc. IEEE/RSJ Int. Conf. Intell. Robots Syst., Sep., 1994, pp. 202-208.

[20] G. Palli and C. Melchiorri, "Model and control of tendon-sheath transmission systems," in Proc. IEEE Int. Conf. Robot. Autom., May 2006, pp. 988-993.

[21] M. Nordin and P. Gutman, "Controlling mechanical systems with backlash-a survey," Automatica, vol. 38, pp. 1633-1649, 2002.

[22] E. K. Bassett, A. H. Slocum, P. T. Masiakos, H. I. Pryor, II, O. C. Farokhzad, and J. M. Karp, "Design of a mechanical clutch-based needleinsertion device," Proc. Nat. Acad. Sci., vol. 106, pp. 5540-5545, 2009.

[23] B. Armstrong-Helouvry, P. E. Dupont, and C. Canudas De Wit, "A survey of analysis tools and compensation methods for control of machines with friction," Automatica, vol. 30, pp. 1083-1138, 1994.

[24] D. A. Recker, P. V. Kokotovic, D. Rhode, and J. Winkelman, "Adaptive nonlinear control of systems containing a deadzone," in Proc. IEEE Conf. Decision Control, Dec. 1991, pp. 2111-2115.

[25] R. Horowitz, "Learning control of robot manipulators," J. Dyn. Syst., Meas., Control, vol. 115, pp. 402-411, 1993.

[26] S. G. Yuen, D. P. Perrin, N. V. Vasilyev, P. J. del Nido, and R. D. Howe, "Force tracking with feed-forward motion estimation for beating heart surgery," IEEE Trans. Robot., vol. 26, no. 5, pp. 888-896, Oct. 2010.

[27] M. Yip, S. G. Yuen, and R. D. Howe, "A robust uniaxial force sensor for minimally invasive surgery," IEEE Trans. Biomed. Eng., vol. 57, pp. 1008-1011, 2010.

[28] S. B. Kesner and R. D. Howe, "Force control of flexible catheter robots for beating heart surgery," in Proc. IEEE Int. Conf. Robot. Autom., Shanghai, China, 2011.

[29] W. J. Book, S. Le, and V. Sangveraphunsiri, "Bracing strategy for robot operation," in Proc. RoManSy 5th CISM-IFToMM Symp., 1984. 
[30] H. West and H. Asada, "A method for the design of hybrid position/Force controllers for manipulators constrained by contact with the environment," in Proc. IEEE Int. Conf. Robot. Autom., Mar. 1985, pp. 251-259.

[31] J. Y. Lew and W. J. Book, "Bracing micro/macro manipulators control," in Proc. IEEE Int. Conf. Robot. Autom., 2011, vol. 3, pp. 2362-2368, May 1994.

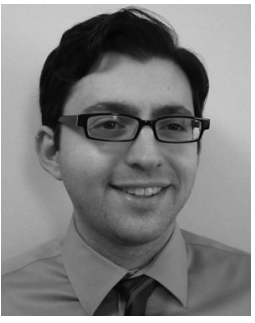

Samuel B. Kesner (M'11) received his bachelors and masters degrees at the Massachusetts Institute of Technology (MIT) in mechanical engineering in 2006 and 2007, respectively. He is currently a senior $\mathrm{Ph} . \mathrm{D}$. candidate in the Biorobotics Lab at the Harvard School of Engineering and Applied Sciences under Prof. Robert Howe where his research focuses on the design of image-guided surgical robots.

His research interests include medical robotics and haptics, bioinspired mechatronics, and the design of medical devices for image-guided and minimally

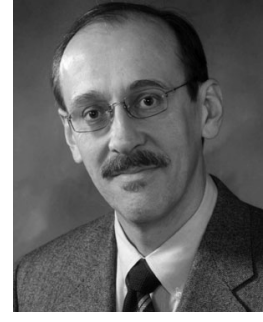

Robert D. Howe (SM'07) is the Abbott and James Lawrence Professor of Engineering, Associate Dean for Academic Programs, and Area Dean for Bioengineering in the Harvard School of Engineering and Applied Sciences. He earned a bachelors degree in physics from Reed College, then worked in the electronics industry in Silicon Valley. He received a doctoral degree in mechanical engineering from Stanford University, then joined the faculty at Harvard in 1990. He directs the Harvard BioRobotics Laboratory, which investigates the roles of sensing and mechanical design in motor control, in both humans and robots.

His research interests focus on manipulation, the sense of touch, haptic interfaces, and robot-assisted and image-guided surgery.

invasive procedures. 